\title{
Gorham-Stout Syndrome with Focal Segmental Glomerulosclerosis: A Case Report
}

\author{
Ji Hyun Kim, M.D. ${ }^{1 *}$ \\ You Sun Kim, M.D. ${ }^{2 *}$ \\ Seon Hee Lim, M.D. ${ }^{2}$ \\ Yo Han Ahn, M.D. ${ }^{2}$ \\ Jung-Min Ko, M.D., Ph.D. ${ }^{2}$ \\ Dong In Suh, M.D., Ph.D. ${ }^{2}$ \\ Kyoung Bun Lee, M.D., Ph.D. ${ }^{3}$ \\ Kyung Chul Moon, M.D., Ph.D. ${ }^{3}$ \\ II-Soo Ha, M.D., Ph.D. ${ }^{2,4}$ \\ Hae ll Cheong, M.D., Ph.D. ${ }^{2,4}$ \\ Hee Gyung Kang, M.D., Ph.D. ${ }^{2,4,5}$
}

Department of Pediatrics', Seoul National University Bundang Hospital, Seongnam, Korea, Departments of Pediatrics ${ }^{2}$, Seoul National University Children's Hospital, Seoul, Korea, Departments of Pathology ${ }^{3}$, Seoul National University Hospital, Seoul, Korea, Kidney Research Institute ${ }^{4}$, Medical Research Center, Seoul National University College of Medicine, Seoul, Korea, Wide River Institute of Immunology ${ }^{5}$, Seoul National University, Seoul, Korea; *These two authors contributed equally to this work.

Corresponding author:

Hee Gyung Kang, M.D., Ph.D.

Division of Pediatric Nephrology, Department of Pediatrics Seoul National University hildren's Hospital \& Seoul National University College of Medicine 101 Daehak-ro, JongnoGu, Seoul 03080, Korea

Tel: +82-2-2072-0658, Fax: +82-2-743-3455

E-mail:kanghg@snu.ac.kr

Received: 27 July 2020

Revised: 27 September 2020

Accepted: 29 September 2020

This is an open-access article distributed under the terms of the Creative Commons Attribution Non-Commercial License (http:// creativecommons.org/licenses/by-nc/4.0/) which permits unrestricted non-commercial use, distribution, and reproduction in any medium, provided the original work is properly cited.
Gorham-Stout syndrome is a rare bone disorder characterized by progressive massive osteolysis and proliferation of vascular and lymphatic vessels. A 15-yearold boy was initially diagnosed with Gorham-Stout at the age of 8 years based on clinical and radiological findings. Following diagnosis, he was treated with pamidronate, interferon alfa, propranolol, oral corticosteroids, and sirolimus. He developed proteinuria at the age of 15 and progressed into the nephrotic range 2 years later. A renal biopsy revealed focal segmental glomerulosclerosis, not otherwise specified variant. The sequential increase in proteinuria associated with medications suggested that the focal segmental glomerulosclerosis may be caused by pamidronate and sirolimus, but cannot completely rule out the possibility of kidney involvement of GSS itself.

Key words: Gorham disease, Osteolysis, Proteinuria, Focal Segmental Glomerulosclerosis, Adolescents

\section{Introduction}

Gorham-Stout Syndrome (GSS) is one of the rare syndromes, also known as vanishing bone or phantom bone disease, characterized by spontaneous and progressive osteolysis. Worldwide, only 200 affected individuals have been reported ${ }^{1}$. Common symptoms include bone pain, swelling, osseous deformity, and pathologic fractures of the extremities. In addition to progressive bone loss commonly involving the clavicles, ribs, and spine, it is also characterized by proliferation of vascular and lymphatic channels in the involved bony segments. It is recognized as an aggressive form of skeletal angiomatosis disease $\mathrm{e}^{2}$. The proliferation of lymphatic vessels may result in chylothorax and pleural effusion. Hardegger et al. ${ }^{3)}$ classified "idiopathic osteolysis" into five classes as follows: type 1, hereditary multicentric carpotarsal osteolysis syndrome (MCTO, OMIM \#166300) with dominant transmission from $M A F B$ gene mutation ${ }^{4}$; type 2 , hereditary multicentric osteolysis with recessive transmission, which is consistent with type 1 in addition to generalized osteoporosis; type 3, nonhereditary multicentric osteolysis with nephropathy; type 4, GSS; and type 5, Winchester syndrome (OMIM \#277950), defined as a monocentric disease of autosomal recessive transmission. Of these, kidney involvement has been reported in type 1 and 3 , and a causative gene was identified only for type 1. For type 4 idiopathic osteolysis, GSS, no genetic cause 
was identified and the etiology remains unclear. Treatment of GSS is largely symptomatic, including surgical treatment, radiotherapy and medications of bisphosphonates, vitamin $\mathrm{D}$, interferon alfa and sirolimus as a novel therapy ${ }^{1}$. Herein we report a case of focal segmental glomerulosclerosis (FSGS) diagnosed in a patient with GSS.

\section{Case report}

A 15-year-old boy with GSS was referred to the Division of Pediatric Nephrology for proteinuria in August 2017. He had recurrent clavicular fractures since the age of 4 and was diagnosed with GSS in 2010, at age 8, based on clinical findings of pleural effusion, recurrent fracture and radiological finding of clavicle osteolysis (Fig. 1). Multiple destructive lesions were noted in the clavicles, multiple ribs, and spine. Pamidronate was administered a total of 20 times either every $1-2$ months $\left(2^{\text {nd }}-19^{\text {th }}\right.$ dose $)$ or intermittently $(0.5-1.5$ year interval) from Oct 2010 to Feb 2016 (Fig. 2) to decrease osteolysis. Pleural effusions recurred intermittently, beginning in February 2015, requiring hospitalization for chest pain and dyspnea. Multiple therapies were administered in an attempt to alleviate the recurrent pleural effusions. Propranolol was administered beginning in March 2015 to decrease VEGF-A levels, interferon alfa was administered from October 2015 to February 2016 for its anti-angiogenic effect and immunity enhancement, and glucocorticosteroids starting in November 2015 and sirolimus beginning in May 2016 to suppress lymphatic proliferation. Although he had experienced respiratory failure several times requiring ventilator care and eventually a tracheostomy, the disease has been under control with these medications with the addition of sirolimus (Fig. 2). Proteinuria was first noted in April 2015, coinciding with an infection and intermittently noted, but was not investigated further until April 2016. Proteinuria was again noted when he was hospitalized for pneumonia in Aug 2017.

On physical examination in Aug 2017, his height was 150 $\mathrm{cm}(<3$ percentile) and weight was $50.1 \mathrm{~kg}(10-25$ percentile). He had normal growth until the age of 12 (height, 2550 percentile; weight, $50-75$ percentile), but experienced growth restriction thereafter. His blood pressure was 124/ $75 \mathrm{mmHg}$ (both $75-90$ percentile), pulse 143 beats/min, respiratory rate 28 breaths/min, body temperature $38.7^{\circ} \mathrm{C}$ and $\mathrm{SpO}_{2} 92 \%$ on room air. Breath sounds were coarse with rales in both lung fields and decreased due to chronic pleural effusion. He urinated well and was not edematous and there was no pitting edema. Laboratory tests revealed hypoalbuminemia (3.0 g/dL; normal, 3.3-5.2 g/dL) and proteinuria (urine protein to creatinine ratio 4.7-10.2; normal, $<0.2)$ (Fig. 2). Serum total protein was low ( $5.8 \mathrm{~g} / \mathrm{dL}$; normal, 6.0-8.0 g/dL), serum electrolytes, blood urea nitrogen, creatinine, total cholesterol, and liver enzymes were all within normal limits. Urinalysis showed intermittent mild microscopic hematuria (5-9 RBC/HPF). Urine beta 2microglobulin and $\mathrm{N}$-acetyl-beta-D-glucosaminidase (NAG)/creatinine ratio were increased to $4.32(0-0.37 \mu \mathrm{g} /$ $\mathrm{mL})$ and $34.2\left(0-5.6 \mathrm{IU} / \mathrm{g}^{\star} \mathrm{Cr}\right)$, respectively. Renal ultrasound showed diffusely increased renal parenchymal echogenicity with a normal kidney size (Fig. 3). Renal biopsy

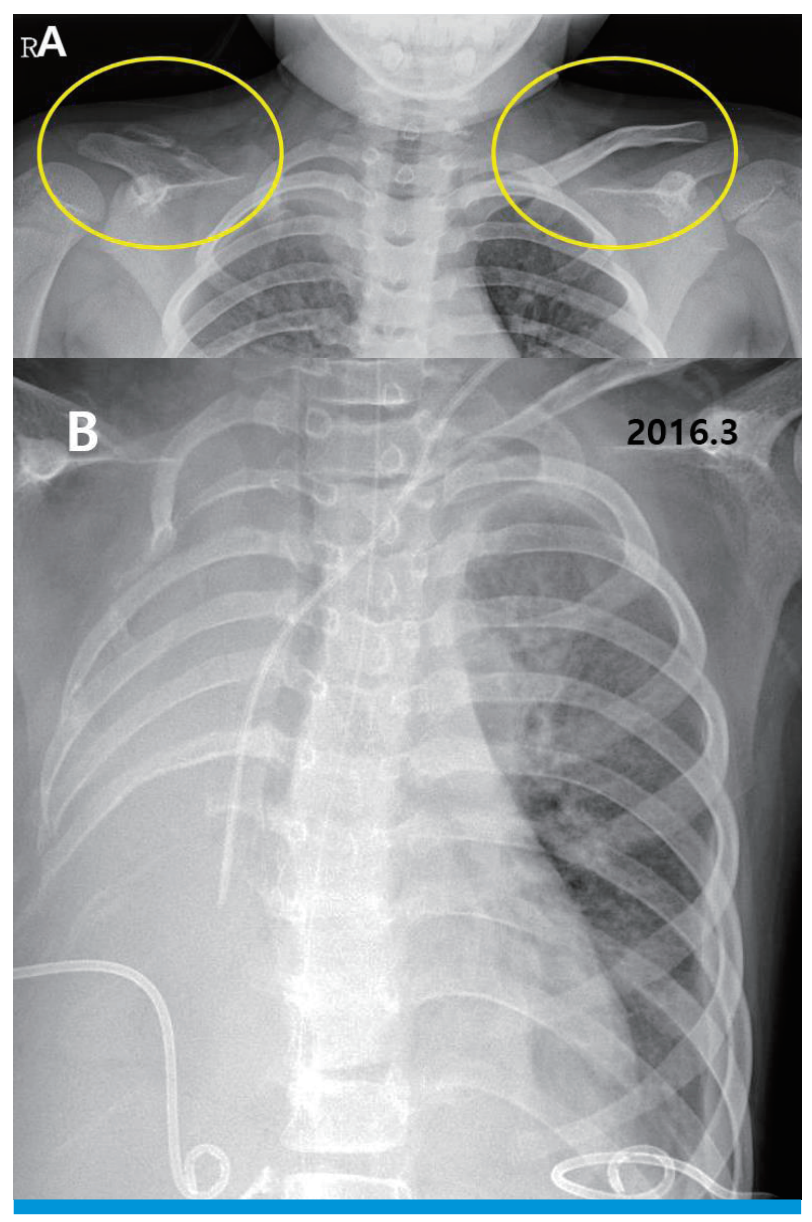

Fig. 1. Radiographic findings. (A) Clavicle, both oblique (Sep 2010), vanishing right clavicle, (B) Chest x-ray (March 2016), severe right pleural effusion with percutaneous catheter drainage bilaterally. 


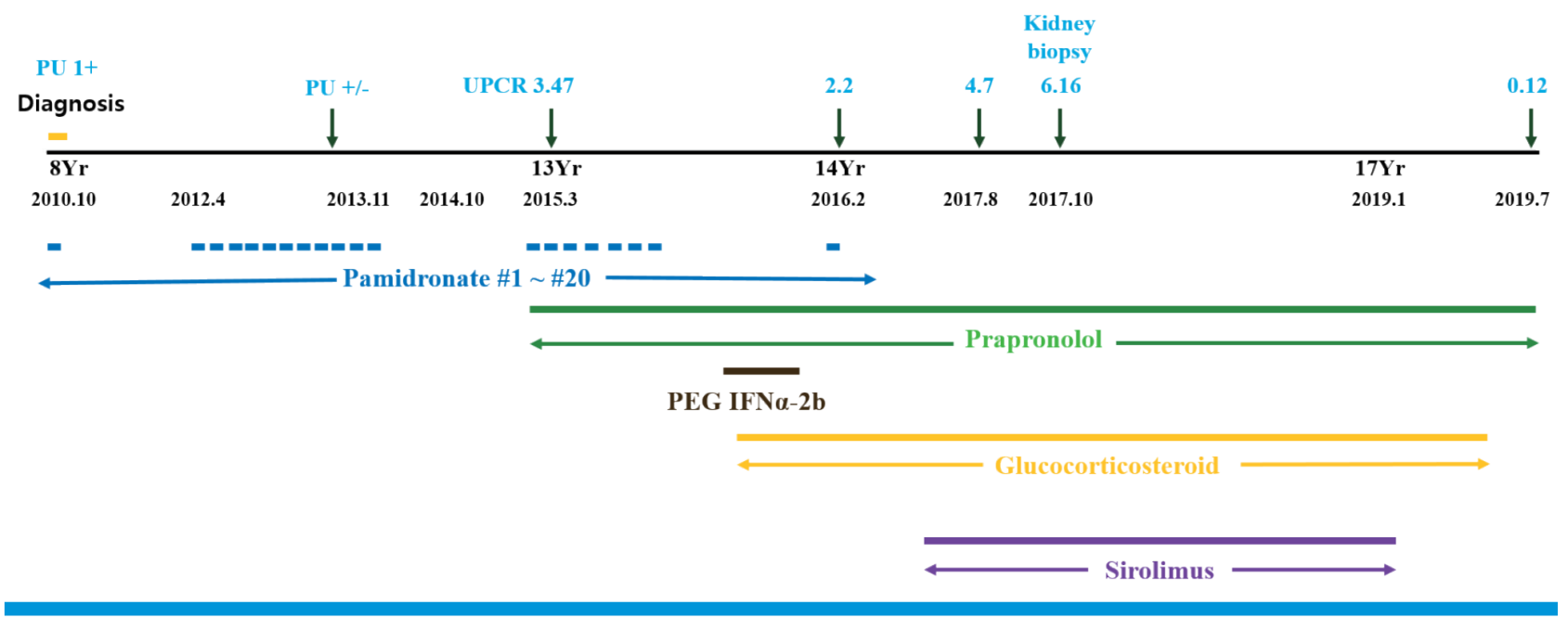

Fig. 2. Treatment history and proteinuria. Yr, year; UPCR, urine protein to creatinine ratio; PU, proteinuria.
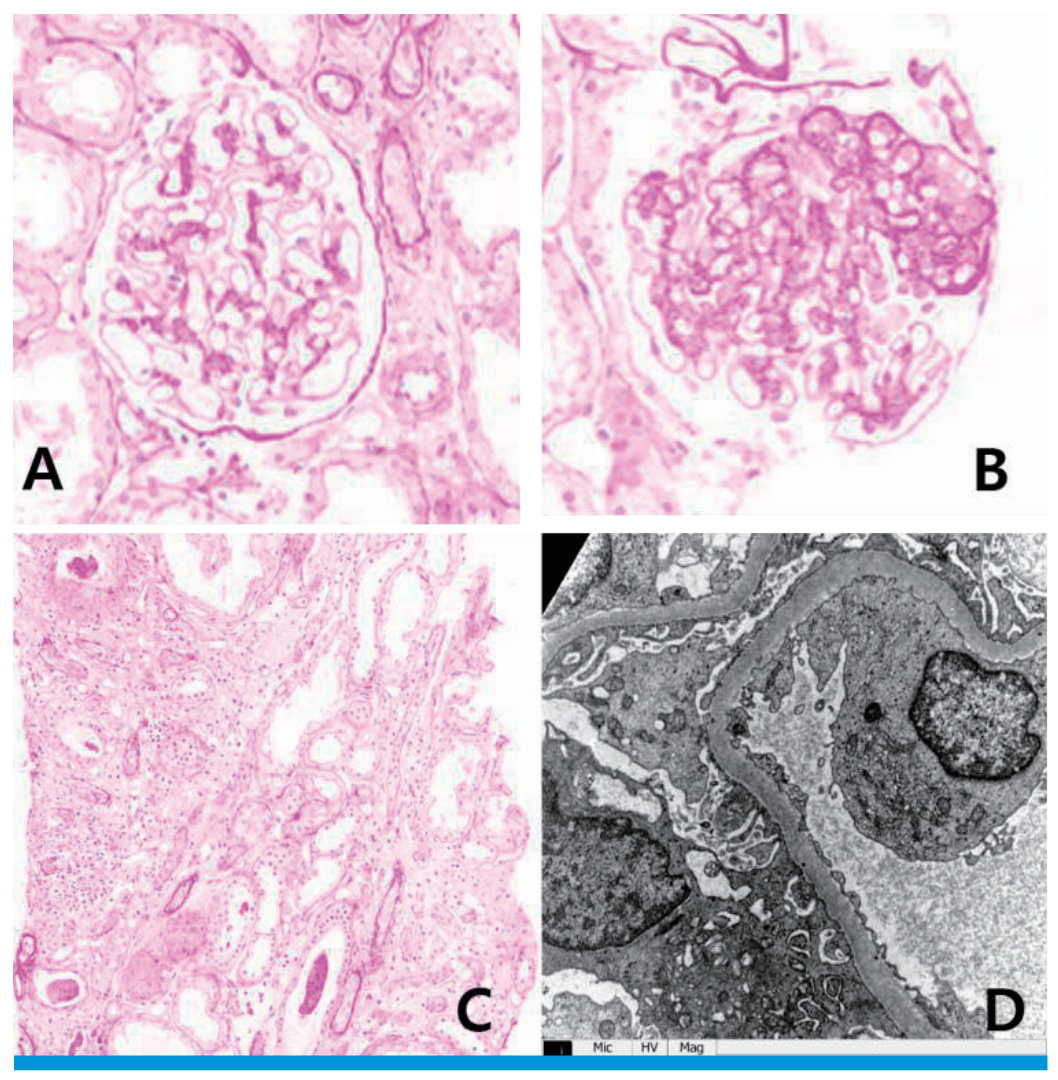

Fig. 3. Light $(A-C)$ and electron (D) microscopic findings of the kidney. $(A)$ Glomerulus of normal size with focal mild hypercellularity involving mesangial cells. (B) Segmental sclerosis (1/14, 7.1\%). (C) Focal mild atrophic tubules and focal mild infiltration of mononuclear cells and eosinophils in the interstitium. (D) Normal glomerular basement membrane with focal mild effacement of the foot processes, and no electron-dense deposits.

was performed in October 2017 and revealed FSGS, not otherwise specified (NOS). Microscopic examination found 1 of 14 (7.1\%) glomeruli with segmental sclerosis and focal mild hypercellularity involving mesangial cells. Focal mild tubular atrophy and injury, focal slight fibrosis, and mild interstitial inflammatory cell (mononuclear cells and 
eosinophils) infiltration was also noted. Immunofluorescence and electron microscopic findings were unremarkable (Fig. 3). Electron microscopic findings showed a normal glomerular basement membrane with mild focal effacement of foot processes. There were no electron-dense deposits.

Given the possibilities of GSS-associated or drug induced glomerulopathy, in order to decrease proteinuria, enalapril, an angiotensin converting enzyme inhibitor was started in March 2017 and was changed to irbesartan, an angiotensin II receptor antagonist, in December 2017. Additionally, sirolimus was tapered over concerns of sirolimus-induced proteinuria. His proteinuria decreased gradually after starting enalapril and reducing the sirolimus, and once irbesartan was initiated, the proteinuria decreased to less than nephrotic range (urine protein to creatinine ratio of 1.64 in April 2018). Serum albumin has remained in the normal range ( $>3.6 \mathrm{~g} / \mathrm{dL}$ ) since November 2017. His GSS is currently well controlled with no more pleural effusions despite the reduction of sirolimus. In January 2019 sirolimus was discontinued. Since then, proteinuria has decreased further and finally disappeared in July 2019. Currently, the disease is well controlled without proteinuria on propranolol and irbesartan.

\section{Discussion}

This case report presents a patient with GSS complicated by FSGS with proteinuria. To our knowledge, there are no reports of an association between GSS and glomerular disease leading to the question of the pathogenesis of FSGS in this patient. Is it an unknown manifestation of GSS itself, or is it secondary to his medications? In fact, pamidronate, sirolimus, and interferon alpha are well known to cause proteinuria and FSGS.

Pamidronate, a bisphosphonate drug, can cause glomerular diseases, such as a collapsing variant of FSGS, or less frequently FSGS NOS or minimal change disease ${ }^{5)}$. It has been linked to proteinuria that usually begins 3 to 42 months after the initiation of therapy ${ }^{6}$. Remission of the glomerular disease following withdrawal of pamidronate persists in many patients; however, some have progressed to end-stage kidney disease despite cessation of the drug ${ }^{7)}$. Barri et al. ${ }^{5}$ suggested that more frequent pamidronate infusion (total number varied from 10 to 40 or more) could be correlated with the incidence of proteinuria and FSGS. In GSS, 6 to over 10 times infusions had been reported ${ }^{8,9)}$. Our patient received pamidronate a total of 20 times given either every 1-2 months or at a $0.5-1.5$ year interval (Fig. 2) and he developed proteinuria 3 years after the initiation of this frequent pamidronate treatment. Additionally, his proteinuria persisted for more than one and a half years following withdrawal of pamidronate. Therefore, it is possible that his proteinuria was initiated by the pamidronate.

The second candidate drug is sirolimus, which is used to treat nephrotic syndrome or proteinuria, but it is known to induce proteinuria in a dose-dependent manner as well. Letavernier et al. ${ }^{10)}$ reported that up to $64 \%$ of patients with chronic allograft nephropathy had massive proteinuria after switching their medication from calcineurin inhibitors (CNI) to sirolimus, and 30\% of them developed FSGS. Moreover, de novo sirolimus glomerular toxicity has also been described in patients who never received $\mathrm{CNI}^{11}$. Besides kidney transplantation, Cho et al. ${ }^{12)}$ reported that sirolimus might exacerbate preexisting idiopathic FSGS resulting in worse proteinuria. Our patient's proteinuria increased into the nephrotic range after the addition of sirolimus and improved in a dose-dependent manner with its reduction (Fig. 4). It is highly likely that sirolimus was associated with the worsening of his proteinuria, whether it caused FSGS or exacerbated preexisting FSGS.

Although interferon alpha is also known to cause FSGS ${ }^{13)}$, it was used only for short time in our patient. Propranolol, another medication used by our patient, has not been reported to cause proteinuria or FSGS. Therefore it is unlikely that his FSGS was caused by either of these two drugs.

Renal dysfunction with osteolysis syndrome syndrome is only known to occur in type 1 (MCTO) and type 3 osteolysis. MCTO from mutation of the $M A F B$ features robust osteoclastogenesis, especially at the carpal and tarsal bones ${ }^{14)}$. The $M A F B$ gene, expressed in glomerular podocytes as well as osteoclasts, plays a crucial role in renal development, and its mutation has been associated with FSGS ${ }^{15)}$. Destructive lesions in the hands and feet which are the characteristics type 1 or 3 osteolysis were not evident in this case. And kidney involvement has not been reported in GSS. Therefore, it is reasonable to assume that this proteinuria was caused by secondary etiology such as medications. In 


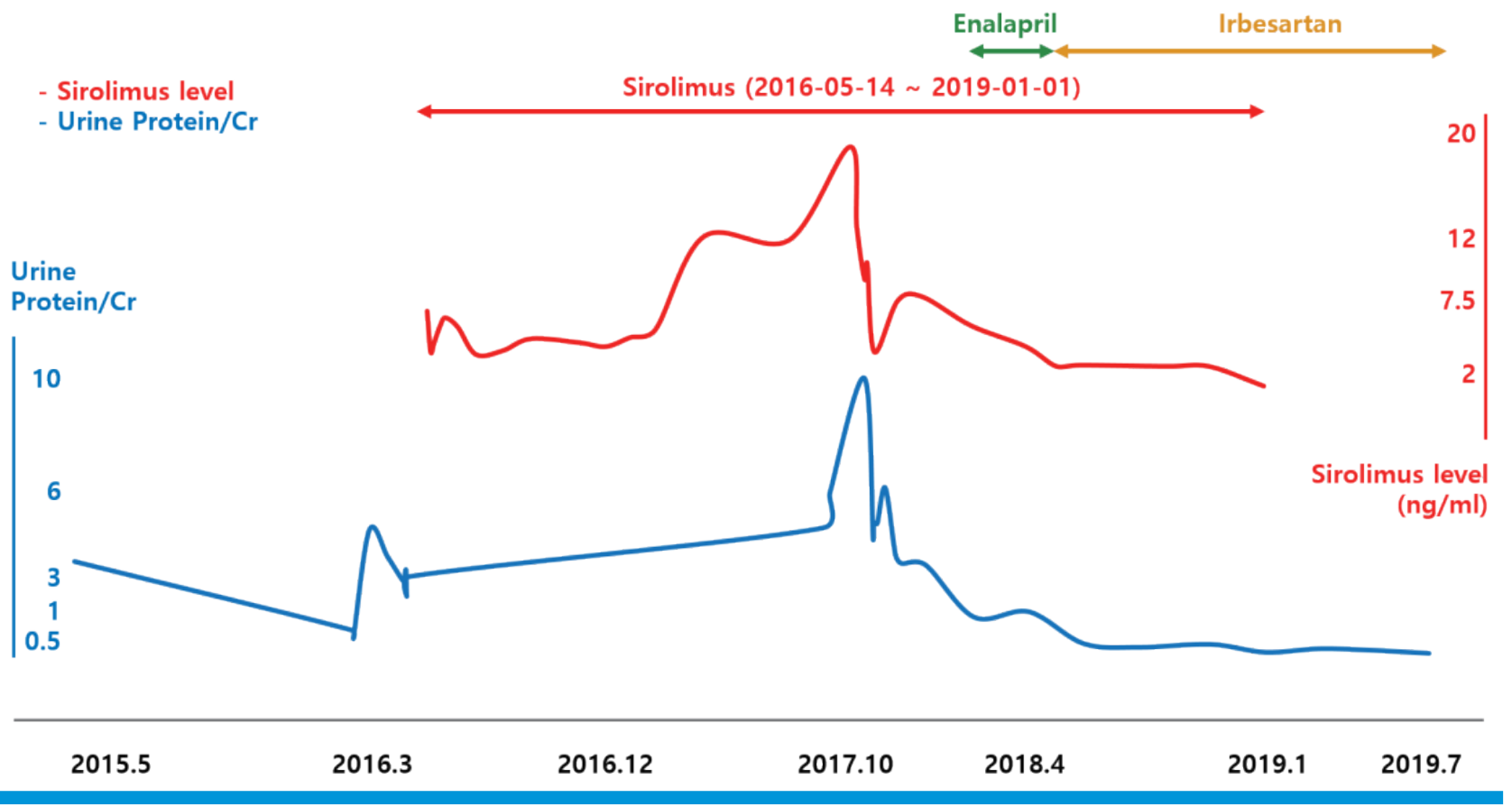

Fig. 4. Relationship among sirolimus levels, renin-angiotensin-aldosterone system inhibitors, and proteinuria.

addition, increased urine beta 2-microglobulin and NAG/ creatinine ratio could be considered to suggest kidney tubular injury, probably caused by certain drugs.

The sequential changes of proteinuria in association with medication changes suggest that the pathogenesis of the proteinuria may be as follows: the proteinuria was initially caused by pamidronate and decreased (or disappeared) following discontinuation of the drug. Sirolimus either aggravated or reinduced proteinuria and FSGS.

In conclusion, we report a rare case of GSS presenting with proteinuria and FSGS which are unexpected findings in GSS. Since FSGS-inducing medications, pamidronate and sirolimus, are indicated for the treatment of osteolysis, it is easy to overlook, but when these medications are used, it is important to monitor the development of proteinuria in this very rare type of vanishing bone syndrome. Nevertheless, we cannot rule out the possibility that this FSGS might be a new findings for GSS.

\section{Acknowledgements}

This research was supported by a grant from the Korea Health Technology R\&D Project through the Korea Health
Industry Development Institute (KHIDI), funded by the Ministry of Health \& Welfare, Republic of Korea (grant number: HI18C0013).

\section{Patient consent}

This study was approved by the institutional review board (IRB), and consent was obtained from the patient and his parent (IRB number H-2003-080-1109).

\section{Conflicts of interest}

No potential conflict of interest relevant to this article was reported.

\section{ORCID IDs}

Ji Hyun Kim https://orcid.org/0000-0001-8477-0157

You Sun Kim https://orcid.org/0000-0002-7687-2687

Seon Hee Lim https://orcid.org/0000-0001-8327-7002

Yo Han Ahn https://orcid.org/0000-0002-8185-4408 
Jung-Min Ko https://orcid.org/0000-0002-0407-7828

Dong In Suh https://orcid.org/0000-0002-7817-8728

Kyoung Bun Lee https://orcid.org/0000-0001-8427-3003

Kyung Chul Moon https://orcid.org/0000-0002-1969-

8360

Il Soo Ha https://orcid.org/0000-0001-5428-6209

Hae-Il Cheong https://orcid.org/0000-0001-7556-1265

Hee Gyung Kang https://orcid.org/0000-0001-8323-

5320

\section{References}

1. Liu Y, Zhong DR, Zhou PR, Lv F, Ma DD, Xia WB, et al. GorhamStout disease: radiological, histological, and clinical features of 12 cases and review of literature. Clin Rheumatol 2016;35:813-23.

2. Al Kaissi A, Scholl-Buergi S, Biedermann R, Maurer K, Hofstaetter JG, Klaushofer K, et al. The diagnosis and management of patients with idiopathic osteolysis. Pediatr Rheumatol 2011;9.

3. Hardegger F, Simpson LA, Segmueller G. The syndrome of idiopathic osteolysis classification, review, and case report. J Bone Joint Surg Br 1985;67:88-93.

4. Zankl A, Duncan EL, Leo PJ, Clark GR, Glazov EA, Addor MC, et al. Multicentric carpotarsal osteolysis is caused by mutations clustering in the amino-terminal transcriptional activation domain of MAFB. Am J Hum Genet 2012;90:494-501.

5. Barri YM, Munshi NC, Sukumalchantra S, Abulezz SR, Bonsib SM, Wallach J, et al. Podocyte injury associated glomerulopathies induced by pamidronate. Kidney Int 2004;65:634-41.
6. Rosenberg AZ, Kopp JB. Focal Segmental Glomerulosclerosis. Clin J Am Soc Nephrol 2017;12:502-17.

7. Perazella MA, Markowitz GS. Bisphosphonate nephrotoxicity. Kidney Int 2008;74:1385-93.

8. Yoon JY, LEE JE, Park SW, Kang MJ, LEE YA, Yang SW et al. Pamidronate Treatment in 9-Year-Old Boy Diagnosed with Gorham Disease. J Korean Soc Pediatr Endocrinol 2011;16:189-92.

9. Hammer F, Kenn W, Wesselmann U, Hofbauer LC, Delling G, Allolio B et al. Gorham-Stout disease - stabilization during bisphosphonate treatment. J Bone Miner Res 2005;20:350-3.

10. Letavernier E, Bruneval P, Mandet C, Duong Van Huyen JP, Peraldi MN, Helal I, et al. High sirolimus levels may induce focal segmental glomerulosclerosis de novo. Clin J Am Soc Nephrol 2007;2:32633.

11. Letavernier E, Bruneval P, Vandermeersch S, Perez J, Mandet C, Belair MF, et al. Sirolimus interacts with pathways essential for podocyte integrity. Nephrol Dial Transplant 2009;24:630-8.

12. Cho ME, Hurley JK, Kopp JB. Sirolimus therapy of focal segmental glomerulosclerosis is associated with nephrotoxicity. Am J Kidney Dis 2007:49:310-7.

13. Markowitz GS, Nasr SH, Stokes MB, D'Agati VD. Treatment with IFN-alpha, -beta, or -gamma is associated with collapsing focal segmental glomerulosclerosis. Clin J Am Soc Nephrol 2010;5: 607-15.

14. Klein C, Bellity J, Finidori G, Glorion C, Pannier S. Multicentric carpotarsal osteolysis syndrome: long-term follow-up of three patients. Skeletal Radiol 2018;47:1015-9.

15. Moriguchi T, Hamada M, Morito N, Terunuma T, Hasegawa K, Zhang C, et al. MAFB Is essential for renal development and F4/80 expression in macrophages. Mol Cell Biol 2006;26:5715-27. 